\title{
Transmitter-Side Antennas Correlation in SVD-Assisted MIMO Systems
}

\author{
Andreas Ahrens ${ }^{1}$, Francisco Cano-Broncano ${ }^{2}$ and César Benavente-Peces ${ }^{2}$ \\ 1 Hochschule Wismar, University of Technology, Business and Design \\ Department of Electrical Engineering and Computer Science \\ Communications Signal Processing Group \\ Philipp-Müller-Straße 14, 23966 Wismar Germany \\ andreas.ahrens@hs-wismar.de \\ http://www.hs-wismar.de \\ 2 Universidad Politécnica de Madrid \\ E.U.I.T de Telecomunicación \\ Ctra. Valencia. km. 7, 28031 Madrid, Spain \\ fcbroncano@gpss. euitt.upm.es \\ cesar.benavente@upm.es \\ http://www.upm.es
}

\begin{abstract}
MIMO techniques allow increasing wireless channel performance by decreasing the BER and increasing the channel throughput and in consequence are included in current mobile communication standards. MIMO techniques are based on benefiting the existence of multipath in wireless communications and the application of appropriate signal processing techniques. The singular value decomposition (SVD) is a popular signal processing technique which, based on the perfect channel state information (PCSI) knowledge at both the transmitter and receiver sides, removes inter-antenna interferences and improves channel performance. Nevertheless, the proximity of the multiple antennas at each front-end produces the so called antennas correlation effect due to the similarity of the various physical paths. In consequence, antennas correlation drops the MIMO channel performance. This investigation focuses on the analysis of a MIMO channel under transmitter-side antennas correlation conditions. First, antennas correlation is analyzed and characterized by the correlation coefficients. The analysis describes the relation between antennas correlation and the appearance of predominant layers which significantly affect the channel performance. Then, based on the SVD, pre- and post-processing is applied to remove inter-antenna interferences. Finally, bit- and power allocation strategies are applied to reach the best performance. The resulting BER reveals that antennas correlation effect diminishes the channel performance and that not necessarily all MIMO layers must be activated to obtain the best performance.
\end{abstract}

Keywords: Multiple-Input Multiple-Output (MIMO) System, Wireless Transmission, Singular Value Decomposition (SVD), Bit Allocation, Power Allocation, Antennas Correlation. 


\section{Introduction}

MIMO communication systems have been studied along the last two decades because their ability to improve wireless channel performance by decreasing the BER and increasing the channel capacity (data rate) without requiring either additional transmit power neither extra bandwidth. In consequence MIMO techniques are incorporated in communication standards. MIMO systems benefits from scattered environments where multipath is present and in order to obtain the full advantages promised by MIMO techniques additional appropriate signal processing techniques are applied to take advantage of the multipath effect [5].

The singular value decomposition (SVD) is a popular technique which allows removing the inter-antenna interferences due to the multiple antennas arrangements at both the transmitter and the receiver sides [3]. The SVD transforms the multipath MIMO channel into multiple independent layers (single-input singleoutput channels, SISO). In order to get the full benefits of using the SVD and obtain the best performance, perfect channel state information (PCSI) should be available at both the transmitter and receiver front-ends. Once the SVD has been applied each resulting layer path is affected by a different gain factor given by the corresponding singular value resulting in layers with different performance. The ideal set-up is that in which after applying the SVD all the layers behave in the same way, i.e., all the singular values take the same value. Unfortunately this is not the common situation and the various layers have different performance.

MIMO wireless channels are affected by the various disturbances influencing regular wireless communication systems. Additionally, due to the use of multiple antennas at both the transmitter and receiver sides, and the typical close spacing of the antennas due to physical limitations, the so called antennas correlation effect arises $[6,10,11]$ affecting the MIMO channel performance.

MIMO channels where antennas are uncorrelated have been largely studied and have reached a state of maturity. In contrast, antennas-correlated MIMO channels require substantial further research in order to characterize the antennas correlation effect and its influence on the channel performance which allows the application of appropriate strategies to optimize the MIMO channel performance.

Antennas correlation diminishes multipath richness, which is essential to MIMO techniques. Due to that effect the various paths established from each transmitter-side antenna to each receiver-side antenna become similar. Under this condition applying the SVD deals to singular values which are quite different and the ratio between the largest and smallest singular values becomes high. In consequence predominant layers arise, some with large singular values which have a pretty good performance and others with quite low singular values which have a poor performance. The overall effect is the drop of the channel performance.

In consequence this paper analyzes and characterizes the antennas correlation effect focusing the attention on the transmitter-side. The analysis remarks the parameters affecting the correlation effect and their influence on the overall 
system performance in order to seek for appropriate strategies to improve the overall channel performance.

Based on independent layers resulting from the application of the pre- and post- processing by using the SVD to the antennas correlated MIMO channel, bit and power allocation techniques can be applied to improve the MIMO system performance $[13,7]$. Given the MIMO channel decomposition into various independent layers with different performance, various transmission modes are defined and investigating by allocating a different number of bits per transmit symbol along the various layers while maintaining the overall data rate. Through the analysis of some exemplary transmission modes this paper shows that not all the layers should be activated in order to obtain the best results.

Power allocation techniques distribute the available transmit power along the different transmit antennas. One of the most popular techniques is the so called water-filling. Based on the layers quality (mainly determined by the corresponding singular value), the layers which should be activated are indentified and hence the total available transmit power is appropriately distributed.

Against the aforementioned background, the novel contribution of this paper is that we demonstrate the benefits of amalgamating a suitable choice of activated MIMO layers and number of bits per symbol together with the appropriate allocation of the transmit power under the constraint of a given fixed data throughput and under the antennas correlation effect. Our results show that under the constraint of correlation only a few number of layers should be used for the data transmission when minimizing the overall bit-error rate.

The remaining part of this paper is organized as follows. Section 2 describes the MIMO system model including the antennas correlation characterization. The bit- and power assignment in correlated channel situation is discussed in section 3 . The obtained results are presented and interpreted in section 4 . Finally section 5 remarks the main results obtained in this investigation.

\section{$2 \quad$ MIMO System Model}

This section is aimed to establish the MIMO system model affected by transmitterside antennas correlation effect. First, the correlation coefficients are defined and characterized in order to finally describe the overall MIMO system model.

\subsection{Transmitter-side correlation coefficients characterization}

The correlation effect is described by the correlation coefficients. Transmitterside antennas correlation coefficients describe the similitude between paths corresponding to a pair of antennas (at the transmitter side) with respect to a reference antenna (at the receiver side). Fig. 1 describes the basic set-up for obtaining the correlation coefficient, where $d$ is the transmitter-side antennas spacing, $d_{1}$ is the distance between transmit antenna \#1 and the receiver-side antenna (taken as reference) and $d_{2}$ is the distance from transmit antenna \#2 to the reference receive antenna (it is assumed $d<<d_{1}, d_{2}$ ); $\phi$ is the departure 
angle. In consequence two paths are described and the correlation coefficient describes how like they are.

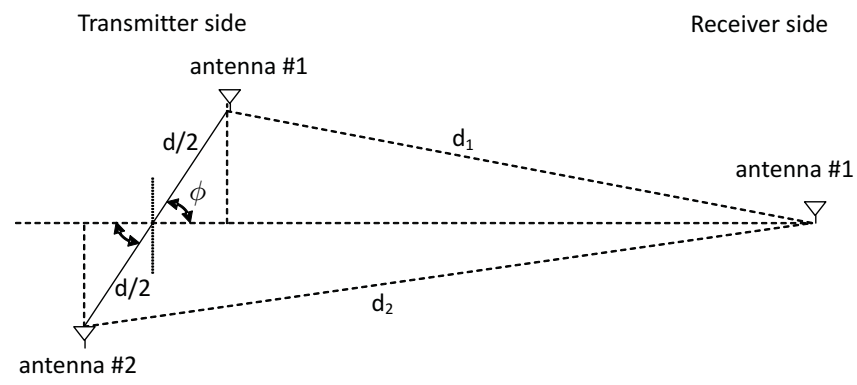

Fig. 1. Antennas' physical disposition: two transmit and one receive antennas

When analyzing the system configuration, presented in Fig. 1, the correlation between the signal $s_{\mathrm{r} 1}(t)$, i. e. the signal arriving at the receive antenna from transmit antenna $\# 1$, and the signal $s_{\mathrm{r} 2}(t)$, i. e. the signal arriving at the receive antenna from transmit antenna \#2, is given by

$$
\rho_{1,2}^{(\mathrm{TX})}=\frac{\mathbf{E}\left\{s_{\mathrm{r} 1}(t) \cdot s_{\mathrm{r} 2}^{*}(t)\right\}-\mathbf{E}\left\{s_{\mathrm{r} 1}(t)\right\} \cdot \mathbf{E}\left\{s_{\mathrm{r} 2}(t)\right\}}{\sqrt{\mathbf{E}\left\{s_{\mathrm{r} 1}(t) \cdot s_{\mathrm{r} 1}^{*}(t)\right\}} \cdot \sqrt{\mathbf{E}\left\{s_{\mathrm{r} 2}(t) \cdot s_{\mathrm{r} 2}^{*}(t)\right\}}},
$$

and simplifies as shown in [2] to

$$
\rho_{1,2}^{(\mathrm{TX})}=\mathrm{e}^{-\mathrm{j} 2 \pi \frac{\left(d_{1}-d_{2}\right)}{\lambda}} .
$$

The transmit antenna separation $d_{\lambda}=d / \lambda$ given in wavelengths units can be expressed as

$$
\frac{d_{2}-d_{1}}{\lambda}=d_{\lambda} \cdot \cos (\phi) \text {. }
$$

Inserting (3) in (2), the transmitter-side correlation coefficient is given by

$$
\rho_{1,2}^{(\mathrm{TX})}=\mathrm{e}^{\mathrm{j} 2 \pi d_{\lambda} \cos (\phi)} .
$$

The antennas path correlation coefficient for line of sight (LOS) trajectories depends on the antennas separation $d_{\lambda}$ and the transmit antennas reference axis rotation angle $\phi$ (or signals angle of departure). By taking the scattered environment of wireless channels into consideration, the transmit antennas reference axis rotation angle $\phi$ becomes time-variant and (4) results in:

$$
\rho_{1,2}^{(\mathrm{TX})}=\mathbf{E}\left\{\mathrm{e}^{\mathrm{j} 2 \pi d_{\lambda} \cos \left(\phi+\xi_{i}\right)}\right\} .
$$

The parameter $\xi_{i}$ in (5) expresses the randomness of the angles for the various scatters and is described by a Gaussian distribution with zero mean and variance 


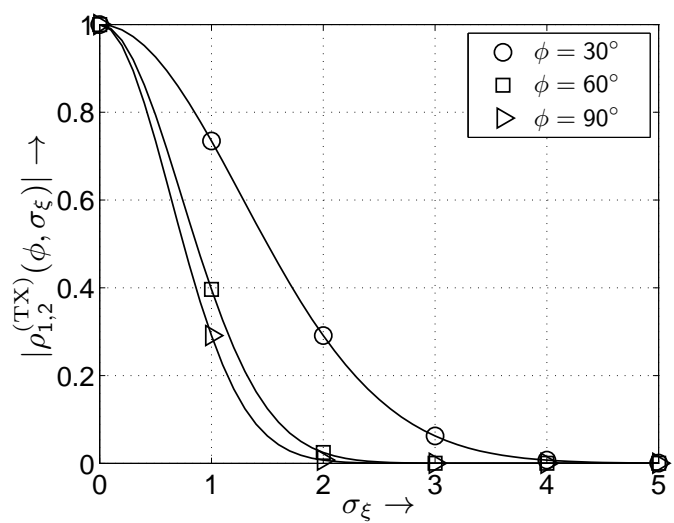

Fig. 2. Variation of the correlation coefficient $\left|\rho_{1,2}^{(\mathrm{TX})}\left(\phi, \sigma_{\xi}\right)\right|$ as a function of $\sigma_{\xi}$ and $\phi$ assuming an antennas separation in wavelengths of $d_{\lambda}=1 / 4$

$\sigma_{\xi}^{2}$. Calculating the expectation under this assumption leads to the following equation:

$$
\rho_{1,2}^{(\mathrm{TX})}\left(\phi, \sigma_{\xi}\right)=\mathrm{e}^{\mathrm{j} 2 \pi d_{\lambda} \cos (\phi)} \mathrm{e}^{-\frac{1}{2}\left(2 \pi d_{\lambda} \sin (\phi) \sigma_{\xi}\right)^{2}} .
$$

When analysing Fig. 2 and Fig. 3, high valued correlation coefficients appear for small values of $\phi$ and $\sigma_{\xi}$ parameters. Additionally, in case of a small antenna separation, i. e. reducing $d_{\lambda}$, the received signals $s_{\mathrm{r} 1}(t)$ and $s_{\mathrm{r} 2}(t)$ become even more similar.

\subsection{Correlated MIMO System Model}

The $\left(n_{\mathrm{R}} \times n_{\mathrm{T}}\right)$ system matrix $\mathbf{H}$ of a correlated MIMO system model is given by

$$
\operatorname{vec}(\mathbf{H})=\mathbf{R}_{\mathrm{HH}}^{\frac{1}{2}} \cdot \operatorname{vec}(\mathbf{G}),
$$

where $\mathbf{G}$ is a $\left(n_{\mathrm{R}} \times n_{\mathrm{T}}\right)$ uncorrelated channel matrix with independent, identically distributed complex-valued Rayleigh elements and $\operatorname{vec}(\cdot)$ is the operator stacking the matrix $\mathbf{G}$ into a vector column-wise [8]. Based on the quite common assumption that the correlation between the antenna elements at the transmitter side is independent from the correlation between the antenna elements at the receiver side, the correlation matrix $\mathbf{R}_{\mathrm{HH}}$ can be decomposed into a transmitter side correlation matrix $\mathbf{R}_{\mathrm{TX}}$ and a receiver side correlation matrix $\mathbf{R}_{\mathrm{RX}}$ following the Kronecker product $\otimes$. Under this assumption the matrix $\mathbf{R}_{\mathrm{HH}}$ is formulated as

$$
\mathbf{R}_{\mathrm{HH}}=\mathbf{R}_{\mathrm{TX}} \otimes \mathbf{R}_{\mathrm{RX}} .
$$

In this paper, no correlation at the receiver side is assumed. Therefore, the $\left(n_{\mathrm{R}} \times n_{\mathrm{R}}\right)$ receiver-side correlation matrix $\mathbf{R}_{\mathrm{RX}}$ simplifies to

$$
\mathbf{R}_{\mathrm{RX}}=\mathbf{I},
$$




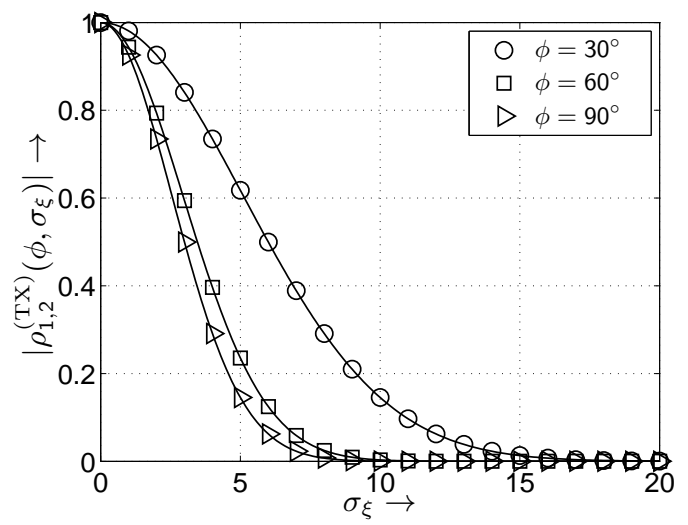

Fig. 3. Variation of the correlation coefficient $\left|\rho_{1,2}^{(\mathrm{TX})}\left(\phi, \sigma_{\xi}\right)\right|$ as a function of $\sigma_{\xi}$ and $\phi$ assuming a wavelength specific antenna separation of $d_{\lambda}=1 / 16$

with the matrix I describing the identity matrix. The $\left(n_{\mathrm{R}} \times n_{\mathrm{R}}\right)$ correlation matrix $\mathbf{R}_{\mathrm{TX}}$ for the investigated $(4 \times 4)$ MIMO system is finally given by:

$$
\mathbf{R}_{\mathrm{TX}}^{(4 \times 4)}=\left(\begin{array}{cccc}
1 & \rho_{1,2}^{(\mathrm{TX})} & \rho_{1,3}^{(\mathrm{TX})} & \rho_{1,4}^{(\mathrm{TX})} \\
\rho_{2,1}^{(\mathrm{TX})} & 1 & \rho_{2,3}^{(\mathrm{TX})} & \rho_{2,4}^{(\mathrm{TX})} \\
\rho_{3,1}^{(\mathrm{TX})} & \rho_{3,2}^{(\mathrm{TX})} & 1 & \rho_{3,4}^{(\mathrm{TX})} \\
\rho_{4,1}^{(\mathrm{TX})} & \rho_{4,2}^{(\mathrm{TX})} & \rho_{4,3}^{(\mathrm{TX})} & 1
\end{array}\right) .
$$

Therein, the correlation coefficient $\rho_{k, \ell}^{(\mathrm{TX})}$ describes the transmitter side correlation between the transmit antenna $k$ and $\ell$. Taking line-of-sight conditions into account, the correlation coefficient results according to (4) in

$$
\rho_{k, \ell}^{(\mathrm{TX})}=\mathrm{e}^{-\mathrm{j} 2 \pi(k-\ell) d_{\lambda} \cos (\phi)} \quad \text { for } \quad k<\ell .
$$

Extending these results to scattered conditions, the transmitter side correlation coefficient results according to (5) for $k<\ell$ in

$$
\rho_{k, \ell}^{(\mathrm{TX})}\left(\phi, \sigma_{\xi}\right)=\mathrm{e}^{-\mathrm{j} 2 \pi(k-\ell) d_{\lambda} \cos (\phi)} \mathrm{e}^{-\frac{1}{2}\left(2 \pi(k-\ell) d_{\lambda} \sin (\phi) \sigma_{\xi}\right)^{2}} .
$$

For the calculation of the transmitter-side correlation coefficient for values of $k>\ell$ it can be exploited that the values of the correlation coefficients are complex conjugated. This is due to the sign change when computing the distance difference between antennas with different antenna reference (see Fig. 4). Finally, the following equation can be used to calculate the correlation coefficient for values of $k>\ell$

$$
\rho_{\ell, k}^{(\mathrm{TX})}=\rho_{k, \ell}^{*(\mathrm{TX})} .
$$

Having frequency non-selective MIMO channels, the whole MIMO system can be decomposed into a number of independent, non-interfering layers. Fig. 5 


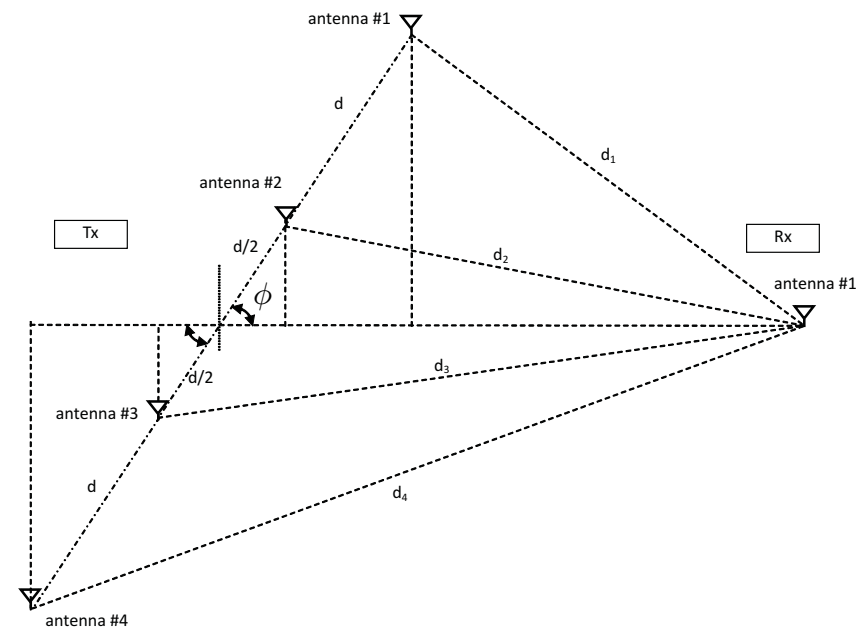

Fig. 4. Antennas' physical disposition: four transmit and one receive antennas

depicts the layer system model resulting after applying the singular value decomposition, where the weighting factors $\sqrt{\xi_{\ell, k}}$ represent the positive square roots of the eigenvalues of the system matrix per MIMO layer $\ell$ and per transmitted data block $k$. The transmitted complex input symbol per MIMO layer $\ell$ is described by $c_{\ell, k}$ and the additive white Gaussian noise (AWGN) by $w_{\ell, k}$, respectively. In general, correlation influenced the unequal weighting of the different

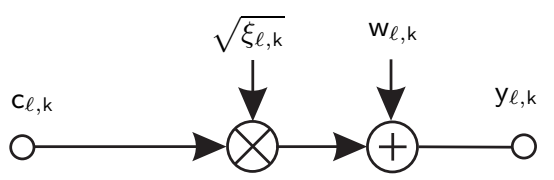

Fig. 5. Resulting system model per MIMO layer $\ell$ and transmitted data block $k$

layers. In order to carefully study the influence of the correlation, two channel constellations are chosen as highlighted in Table 1. The corresponding unequal weighting of the different layers is shown in Fig. 6 and Fig. 7 for an exemplarily studied $(4 \times 4)$ MIMO system. Therein, the difference in the layer-specific fluctuations is described by the probability density function (pdf) of the parameter $\vartheta=\sqrt{\xi_{\ell=4, k}} / \sqrt{\xi_{\ell=1, k}}$, which shows the unequal weighting of the different layers within the MIMO system.

The ratio between the smallest and the largest singular values is an appropriate way to infer the antennas correlation effects. When this parameter $\vartheta$ approaches the unity the MIMO channel is close to the best performance 
Table 1. Parameters of the of the investigated channel constellations

\begin{tabular}{cccc}
\hline Description & $\phi$ & $\sigma_{\xi}$ & $d_{\lambda}$ \\
\hline \hline Weak correlation & $30^{\circ}$ & 1 & 1 \\
\hline Strong correlation & $30^{\circ}$ & 1 & 0,25 \\
\hline \hline
\end{tabular}

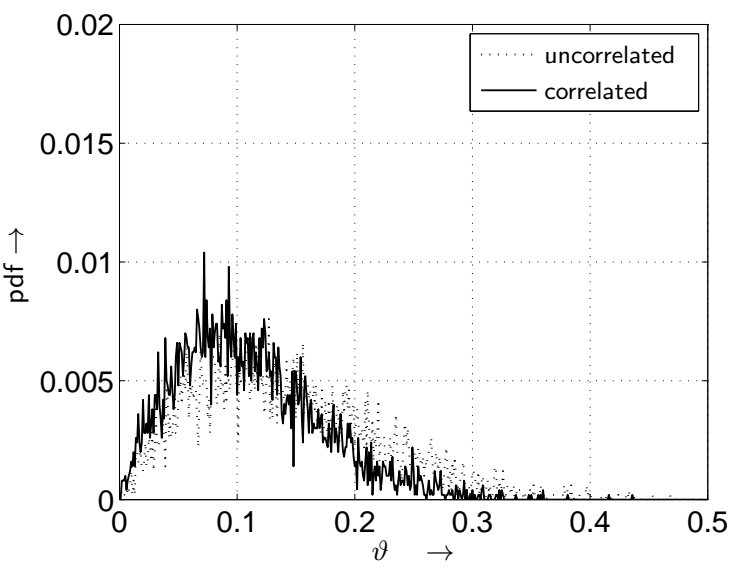

Fig. 6. PDF (probability density function) of the ratio $\vartheta$ between the smallest and the largest singular value for weakly correlated (solid line) as well as uncorrelated (dotted line) frequency non-selective $(4 \times 4)$ MIMO channels $\left(d_{\lambda}=1, \phi=30^{\circ}\right.$ and $\left.\sigma_{\xi}=1,0\right)$

which is reached when all the layers have the same performance (assuming the same noise power at the receiver-side). In this particular case the layer-specific weighting factors, i. e., the singular values, are very similar. For weak antennas correlation, as depicted in Fig. 6, this parameter decreases which means some layers performs better than others and the overall MIMO channel performance drops. When antennas correlation is significantly high, the parameter $\vartheta$ becomes smaller, meaning a noticeable difference in the performance of the various layer. Now, predominant strong and weak layers appear which decreases the overall channel performance (by increasing the BER).

The distribution of the layer-specific characteristic can be studied when analyzing the CCDF (complementary cumulative distribution function) for the different degrees of correlation as shown in Fig. 8 and Fig. 9. The antennas correlation increases the probability of having layers with larger values (see layers $\sqrt{\xi_{1}}$ and $\sqrt{\xi_{2}}$ ) and increases for weak layers the probability of having lower values (see layers $\sqrt{\xi_{3}}$ and $\sqrt{\xi_{4}}$ ).

The inspection of the CCDF also provides relevant information to predict the MIMO channel performance. Increasing antennas correlation spreads the singular values CCDF curves. For the non-correlated case, the various layers CCDF seem to be more concentrated. In the ideal case all of them overlap and the best 


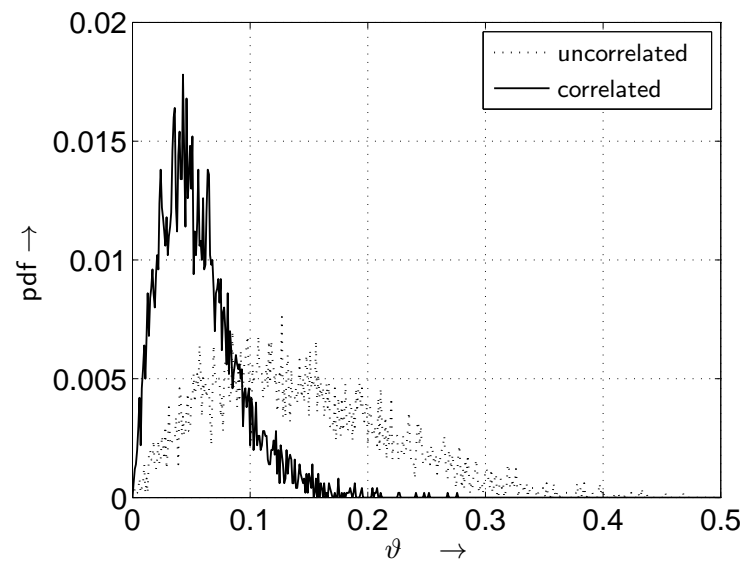

Fig. 7. PDF (probability density function) of the ratio $\vartheta$ between the smallest and the largest singular value for strongly correlated (solid line) as well as uncorrelated (dotted line) frequency non-selective $(4 \times 4)$ MIMO channels $\left(d_{\lambda}=1 / 4, \phi=30^{\circ}\right.$ and $\left.\sigma_{\xi}=1,0\right)$

performance is obtained. Weak correlation spreads the curves by right shifting those corresponding to the highest singular values increasing the probability of getting large values in contrast to the smallest singular values. Under strong antennas correlation the CCDF curve for the largest singular values are indeed more right shifted while the smallest ones are left shifted. In consequence, in this case the probability of the largest singular value to obtain a high value increases while the probability of taking the smallest singular values a lower value also increases dealing to the MIMO channel worse performance.

\section{Bit- and Power Assignment}

Assuming $M$-ary Quadrature Amplitude Modulation (QAM), the argument $\varrho=$ $U_{\mathrm{A}}^{2} / U_{\mathrm{R}}^{2}$ of the complementary error function $[4,9]$ can be used to optimize the quality of a data communication system by taking the half-vertical eye-opening $U_{\mathrm{A}}$ and the noise power per quadrature component $U_{\mathrm{R}}^{2}$ at the detector input into account [1]. The half-vertical eye-opening per MIMO layer $\ell$ and per transmitted symbol block $k$ results in

$$
U_{\mathrm{A}}^{(\ell, k)}=\sqrt{\xi_{\ell, k}} \cdot U_{\mathrm{s} \ell},
$$

where $U_{\mathrm{s} \ell}$ denotes the half-level transmit amplitude assuming $M_{\ell \text {-ary }}$ QAM and $\sqrt{\xi_{\ell, k}}$ represents the positive square roots of the eigenvalues of the matrix $\mathbf{H}^{\mathrm{H}} \mathbf{H}$. The average transmit power $P_{\mathrm{s} \ell}$ per MIMO layer $\ell$ determines the halflevel transmit amplitude $U_{\mathrm{s} \ell}$ and is given by

$$
P_{\mathrm{s} \ell}=\frac{2}{3} U_{\mathrm{s} \ell}^{2}\left(M_{\ell}-1\right) \text {. }
$$




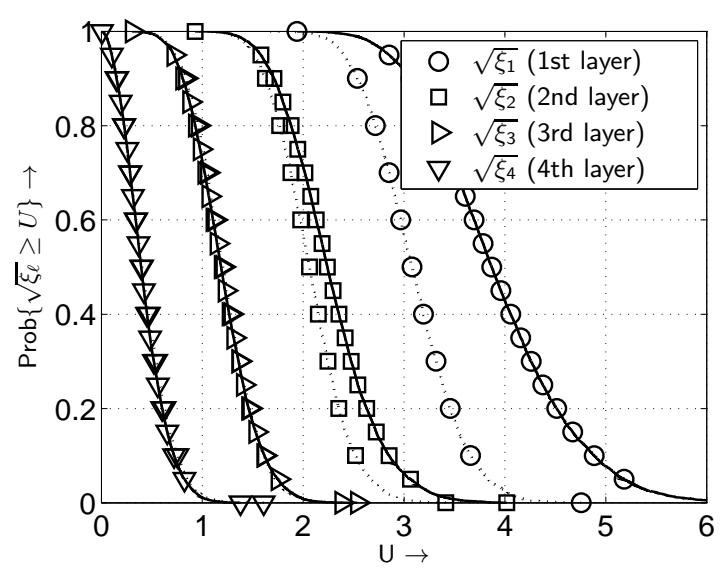

Fig. 8. CCDF of the layer-specific distribution for weakly correlated (solid line) as well as uncorrelated (dotted line) frequency non-selective $(4 \times 4)$ MIMO channels $\left(d_{\lambda}=1\right.$, $\phi=30^{\circ}$ and $\left.\sigma_{\xi}=1,0\right)$

Activating $L \leq \min \left(n_{\mathrm{T}}, n_{\mathrm{R}}\right)$ MIMO layers, the overall transmit power $P_{\mathrm{s}}=$ $\sum_{\ell=1}^{L} P_{\mathrm{s} \ell}$ can be calculated.

Power Allocation (PA) can be used to balance the BER in the different numbers of activated MIMO layers [1]. The resulting layer-specific system model including power allocation is highlighted in Fig. 10. The layer-specific power allocation factors $\sqrt{p_{\ell, k}}$ adjust the half-vertical eye opening according to

$$
U_{\mathrm{APA}}^{(\ell, k)}=\sqrt{p_{\ell, k}} \cdot \sqrt{\xi_{\ell, k}} \cdot U_{\mathrm{s} \ell} .
$$

This results in the layer-specific transmit power per symbol block $k$

$$
P_{\mathrm{sPA}}^{(\ell, k)}=p_{\ell, k} P_{\mathrm{s} \ell}
$$

Taking all activated MIMO layers into account, the overall transmit power per symbol block $k$ is obtained as

$$
P_{\mathrm{sPA}}^{(k)}=\sum_{\ell=1}^{L} P_{\mathrm{sPA}}^{(\ell, k)}=P_{\mathrm{s}} .
$$

In order to balance the BER in the different numbers of activated MIMO layers, solutions for the so far unknown PA parameters are needed.

A simplified PA solution can be found when guaranteeing that the signal-tonoise-ratio at the detector input is the same for all activated MIMO layers per data block $k$. In this particular case, the following condition should be ensured 


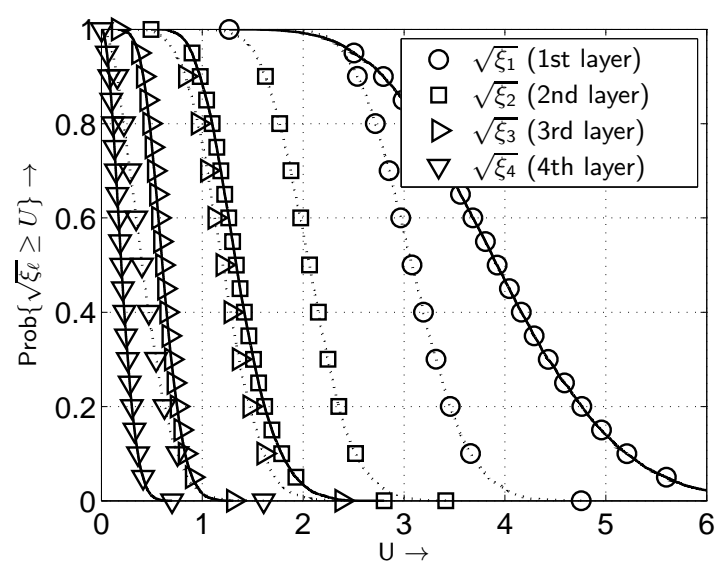

Fig. 9. CCDF of the layer-specific distribution for strongly correlated (solid line) as well as uncorrelated (dotted line) frequency non-selective $(4 \times 4)$ MIMO channels $\left(d_{\lambda}=1 / 4\right.$, $\phi=30^{\circ}$ and $\left.\sigma_{\xi}=1,0\right)$

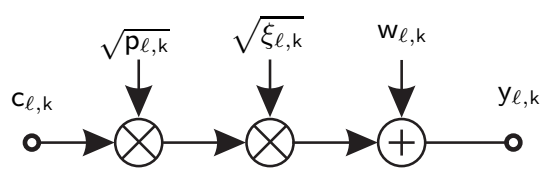

Fig. 10. Resulting layer-specific system model including MIMO-layer PA

for the signal-to-noise ratio at the detector input

$$
\varrho_{\mathrm{PA}}^{(\ell, k)}=\frac{\left(U_{\mathrm{A} \mathrm{PA}}^{(\ell, k)}\right)^{2}}{U_{\mathrm{R}}^{2}}=\text { constant } \quad \ell=1,2, \ldots, L .
$$

When assuming an identical detector input noise variance $U_{\mathrm{R}}^{2}$ for each channel output symbol the beforehand introduces Equal-SNR criteria requires the same half vertical eye opening of each channel output symbol

$$
U_{\mathrm{APA}}^{(\ell, k)}=\mathrm{constant} \quad \ell=1,2, \ldots, L
$$

The power to be allocated to each activated MIMO layer $\ell$ and transmitted data block $k$ can be shown to be calculated as follows:

$$
p_{\ell, k}=\frac{1}{U_{\mathrm{s} \ell}^{2} \cdot \xi_{\ell, k}} \cdot \frac{L}{\sum_{\nu=1}^{L} \frac{1}{U_{\mathrm{s} \nu}^{2} \cdot \xi_{\nu, k}}},
$$


Table 2. Investigated QAM transmission modes

\begin{tabular}{ccccc}
\hline \multicolumn{5}{c}{ throughput layer 1 layer 2 layer 3 layer 4} \\
\hline $8 \mathrm{bit} / \mathrm{s} / \mathrm{Hz}$ & 256 & 0 & 0 & 0 \\
$\mathbf{8} \mathrm{bit} / \mathrm{s} / \mathbf{H z}$ & $\mathbf{6 4}$ & $\mathbf{4}$ & $\mathbf{0}$ & $\mathbf{0}$ \\
$\mathbf{8} \mathrm{bit} / \mathrm{s} / \mathbf{H z}$ & $\mathbf{1 6}$ & $\mathbf{1 6}$ & $\mathbf{0}$ & $\mathbf{0}$ \\
$\mathbf{8} \mathrm{bit} / \mathrm{s} / \mathbf{H z}$ & $\mathbf{1 6}$ & $\mathbf{4}$ & $\mathbf{4}$ & $\mathbf{0}$ \\
$8 \mathrm{bit} / \mathrm{s} / \mathrm{Hz}$ & 4 & 4 & 4 & 4 \\
\hline
\end{tabular}

and guarantees for each channel output symbol $(\ell=1, \ldots, L)$ the same half vertical eye opening of

$$
U_{\mathrm{APA}}^{(\ell, k)}=\sqrt{p_{\ell, k}} \cdot \sqrt{\xi_{\ell, k}} \cdot U_{\mathrm{s} \ell}=\sqrt{\frac{L}{\sum_{\nu=1}^{L} \frac{1}{U_{\mathrm{s} \nu}^{2} \xi_{\nu, k}}}} .
$$

Together with the identical detector input noise variance for each channel output symbol, the above-mentioned equal quality scenario is encountered.

\section{Results}

In this work a $(4 \times 4)$ MIMO system with transmitter-side antennas correlation is studied.

In order to transmit at a fixed data rate while maintaining the best possible integrity, i. e., bit-error rate, an appropriate number of MIMO layers has to be used, which depends on the specific transmission mode, as detailed in Table 1.

The choice of fixed transmission modes regardless of the channel quality can be justified when analyzing the probability of choosing a specific transmission mode by using optimal bit loading [12]. As highlighted in Table 3 for uncorrelated MIMO channels, it turns out that only an appropriate number of MIMO layers has to be activated, e.g., the $(16,4,4,0)$ QAM configuration. However, when

Table 3. Probability of choosing specific transmission modes at a fixed data rate by using optimal bitloading $\left(10 \cdot \log _{10}\left(E_{\mathrm{s}} / N_{0}\right)=10 \mathrm{~dB}\right)$

\begin{tabular}{ccccc}
\hline mode & $(64,4,0,0)$ & $(16,16,0,0)$ & $(16,4,4,0)$ & $(4,4,4,4)$ \\
\hline pdf & 0.0116 & 0.2504 & 0.7373 & 0.0008 \\
\hline
\end{tabular}

the correlation effect appears as illustrated in Table 4 for weakly correlated as 
Table 4. Probability of choosing specific transmission modes in weakly correlated MIMO channels at a fixed data rate by using optimal bitloading $\left(10 \cdot \log _{10}\left(E_{\mathrm{s}} / N_{0}\right)=10\right.$ $\mathrm{dB})$

\begin{tabular}{ccccc}
\hline mode & $(64,4,0,0)$ & $(16,16,0,0)$ & $(16,4,4,0)$ & $(4,4,4,4)$ \\
\hline pdf & 0.1274 & 0.3360 & 0.5366 & 0.0 \\
\hline
\end{tabular}

Table 5. Probability of choosing specific transmission modes in strongly correlated MIMO channels at a fixed data rate by using optimal bitloading $\left(10 \cdot \log _{10}\left(E_{\mathrm{s}} / N_{0}\right)=10\right.$ $\mathrm{dB})$

\begin{tabular}{ccccc}
\hline mode & $(64,4,0,0)$ & $(16,16,0,0)$ & $(16,4,4,0)$ & $(4,4,4,4)$ \\
\hline pdf & 0.8252 & 0.1087 & 0.0605 & 0.0 \\
\hline
\end{tabular}

well as in Table 5 for highly correlated MIMO channels, the importance of using layers with large singular values increases.

The optimal performance results when using PA are shown in Fig. 11 and Fig. 12: The BER becomes minimal in case of an optimized bit loading with highest bit loading in the layer with largest singular values.

Fig. 13 and 14 show the MIMO system performance when using fixed transmission modes and having different degrees of correlation. As highlighted by the BER curves, in case of high correlation only the layers with the largest singular values should be used for the data transmission.

\section{Conclusion}

This paper has shown the characterization of the correlation coefficients in a MIMO channel with transmitter-side antennas correlation has analyzed the MIMO channel performance under correlation. The correlation coefficients depend on paths physical parameters describing the antennas arrangement. Antennas spacing, angle of departure and the scattering angle affect the paths correlation degree and hence the MIMO performance. The singular value decomposition (SVD) is a signal processing technique that having perfect channel state information at both the transmit and receive sides involves pre- and postprocessing at the transmit and receive sides respectively which converts the MIMO channel into independent layers characterized by the layer gain given by the singular values. Antennas correlation affects the SVD dealing to dispersed singular values: some with large values and others with low values, in contrast to the ideal case. This singular values dispersion yields a poor MIMO channel performance due to those layers with low singular values which present a low reliability. The overall antennas correlation effect is described by the correlation matrix which relates the channel uncorrelated matrix to the correlated one. 


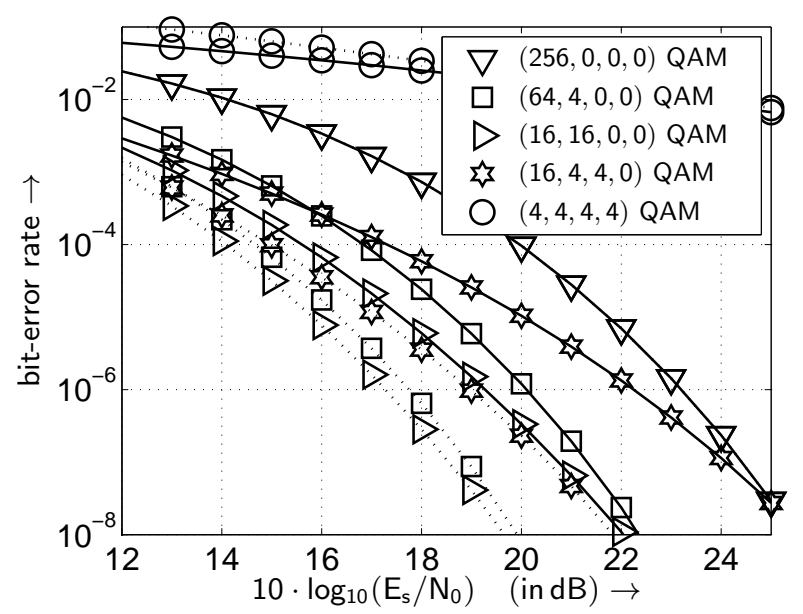

Fig. 11. BER with optimal PA (dotted line) and without PA (solid line) when using the transmission modes introduced in Tab. 2 and transmitting $8 \mathrm{bit} / \mathrm{s} / \mathrm{Hz}$ over frequency non-selective $(4 \times 4)$ MIMO channels $\left(d_{\lambda}=1, \phi=30^{\circ}\right.$ and $\left.\sigma_{\xi}=1,0\right)$ with weak transmitter-side correlation

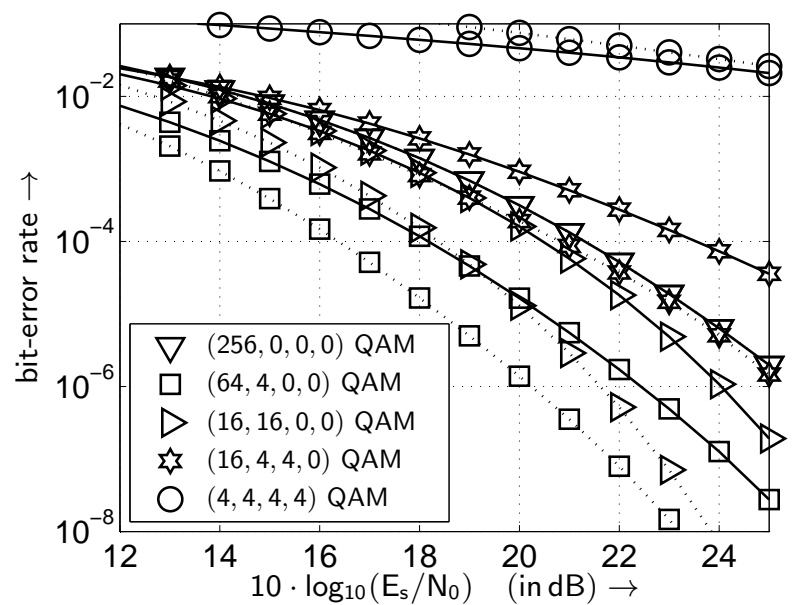

Fig. 12. BER with optimal PA (dotted line) and without PA (solid line) when using the transmission modes introduced in Tab. 2 and transmitting $8 \mathrm{bit} / \mathrm{s} / \mathrm{Hz}$ over frequency non-selective $(4 \times 4)$ MIMO channels $\left(d_{\lambda}=1 / 4, \phi=30^{\circ}\right.$ and $\left.\sigma_{\xi}=1,0\right)$ with strong transmitter-side correlation 


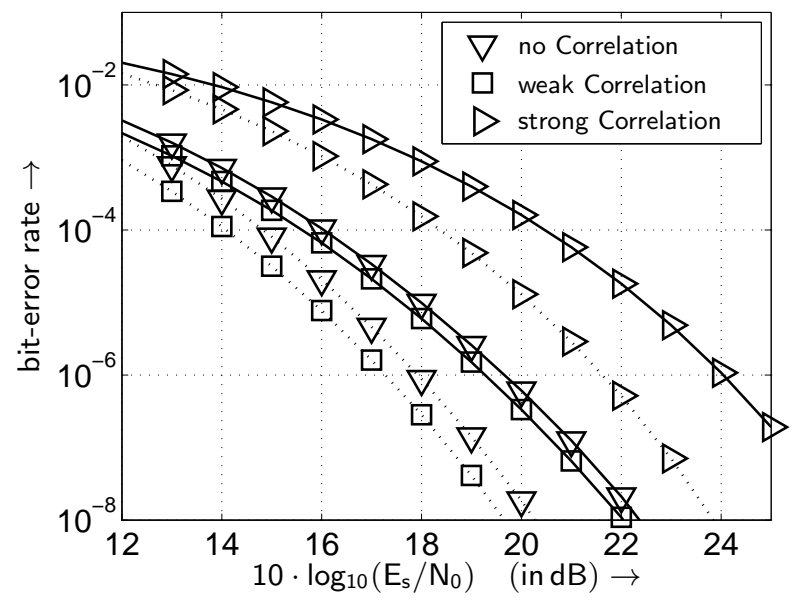

Fig. 13. BER with optimal PA (dotted line) and without PA (solid line) when using the $(16,16,0,0$ QAM transmission mode and transmitting $8 \mathrm{bit} / \mathrm{s} / \mathrm{Hz}$ over frequency non-selective $(4 \times 4)$ MIMO channels with different degrees of correlation

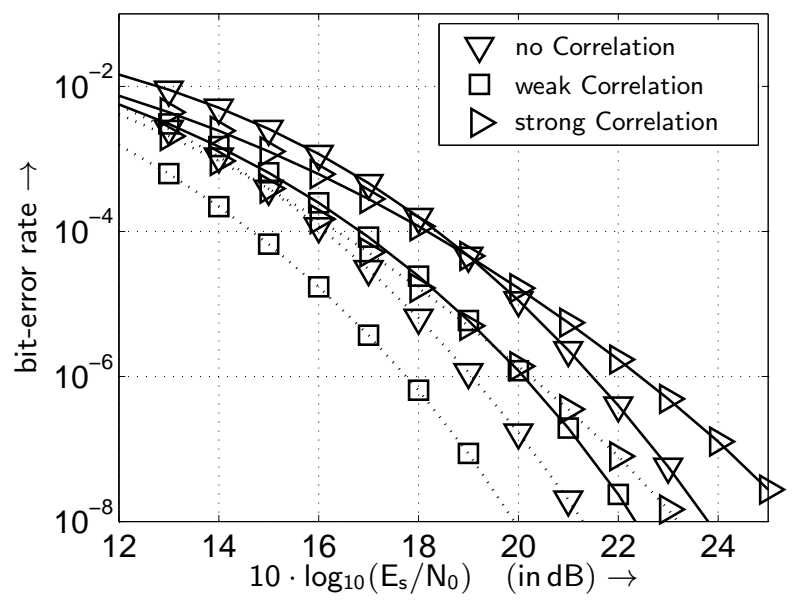

Fig. 14. BER with optimal PA (dotted line) and without PA (solid line) when using the $(64,4,0,0$ QAM transmission mode and transmitting $8 \mathrm{bit} / \mathrm{s} / \mathrm{Hz}$ over frequency non-selective $(4 \times 4)$ MIMO channels with different degrees of correlation 
The analysis has demonstrated that the representation of the PDF of the ratio between the smallest and the largest singular values provides a useful mean to predict the channel behavior and the appropriateness of activating all the MIMO layers. Besides, the singular values CCDF gives relevant information concerning the probability of obtaining predominant (strong and weak) layers and infer the MIMO channel behaviour. The best performance is obtained when all CCDF coincide (are the same) or are quite close. CCDF curve dispersion reveals the existence of predominant layer lowering the MIMO performance. Additionally, in order to mitigate correlation effects the investigation has analyzed the effect of bit and transmit power allocation along the various MIMO layers as techniques for improving channel performance even in the presence of antennas correlation. Regarding the power allocation, a basic technique has been applied in order to obtain the same quality along the different activated layers, i.e., the same SNR at each detector. This technique allows obtaining a higher performance. Moreover, bit loading has been studied through the description of some profiles (transmission modes) dealing to different constellation per layer (bit per symbol interval) but maintaining the overall transmission rate. A remarkable conclusion is that activating all the MIMO layers not necessarily provides the best performance as highlighted in the results where the transmission modes $(64,4,0,0)$ and $(16,16,0,0)$ present the best performance. In order to highlight the importance of this fact the probability of using each transmission mode was analyzed and the previous conclusion was remarked.

\section{References}

1. Ahrens, A. and Lange, C. (2008). Modulation-Mode and Power Assignment in SVD-equalized MIMO Systems. Facta Universitatis (Series Electronics and Energetics), 21(2):167-181.

2. Cano-Broncano, F., Benavente-Peces, C., Ahrens, A., Ortega-Gonzalez, F. J., and Pardo-Martin, J. M. (2013). Analysis of MIMO Systems with Transmitter-Side Antennas Correlation. In International Conference on Pervasive and Embedded Computing and Communication Systems (PECCS), Barcelona (Spain).

3. Haykin, S. S. (2002). Adaptive Filter Theory. Prentice Hall, New Jersey.

4. Kalet, I. (1987). Optimization of Linearly Equalized QAM. IEEE Transactions on Communications, 35(11):1234-1236.

5. Kühn, V. (2006). Wireless Communications over MIMO Channels - Applications to CDMA and Multiple Antenna Systems. Wiley, Chichester.

6. Lee, W.-Y. (1973). Effects on Correlation between two Mobile Radio Base-Station Antennas. IEEE Transactions on Vehicular Technology, 22(4):130-140.

7. Mutti, C. and Dahlhaus, P. (2004). Adaptive Power Loading for Multiple-Input Multiple-Output OFDM Systems with Perfect Channel State Information. In Joint COST 273/284 Workshop on Antennas and Related System Aspects in Wireless Communications, pages 93-98, Gothenburg.

8. Oestges, C. (2006). Validity of the Kronocker Model for MIMO Correlated Channels. In Vehicular Technology Conference, volume 6, pages 2818-2822, Melbourne.

9. Proakis, J. G. (2000). Digital Communications. McGraw-Hill, Boston. 
10. Salz, J. and Winters, J. H. (1994). Effect of Fading Correlation on adaptive Arrays in digital Mobile Radio. IEEE Transactions on Vehicular Technology, 43(4):10491057.

11. Shiu, D., Foschini, G., Gans, M., and Kahn, J. (2000). Fading Correlation and its Effect on the Capacity of Multielement Antenna Systems. IEEE Transactions on Communications, 48(3):502-513.

12. Wong, C. Y., Cheng, R. S., Letaief, K. B., and Murch, R. D. (1999). Multiuser OFDM with Adaptive Subcarrier, Bit, and Power Allocation. IEEE Journal on Selected Areas in Communications, 17(10):1747-1758.

13. Zhou, Z., Vucetic, B., Dohler, M., and Li, Y. (2005). MIMO Systems with Adaptive Modulation. IEEE Transactions on Vehicular Technology, 54(5):1073-1096. 